\title{
Anti-Kondo resonance in transport through a quantum wire with a side-coupled quantum dot
}

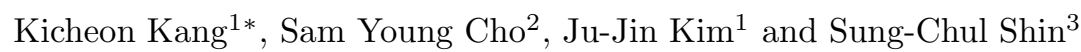 \\ ${ }^{1}$ Department of Physics, Chonbuk National University, Chonju 561-756, Chonbuk, Korea \\ ${ }^{2}$ Institute of Physics and Applied Physics, Yonsei University, Seoul 120-749, Korea \\ ${ }^{3}$ Department of Physics, Korea Advanced Institute of Science and Technology, Taejon 305-701, Korea
}

(October 26, 2018)

\begin{abstract}
An interacting quantum dot side-coupled to a perfect quantum wire is studied. Transport through the quantum wire is investigated by using an exact sum rule and the slave-boson mean field treatment. It is shown that the Kondo effect provides a suppression of the transmission due to the destructive interference of the ballistic channel and the Kondo channel. At finite temperatures, anti-resonance behavior is found as a function of the quantum dot level position, which is interpreted as a crossover from the high temperature Kondo phase to the low temperature charge fluctuation phase.
\end{abstract}

PACS numbers: 72.15.Qm, 73.23.Ad, 73.40.Gk

The Kondo effect has been a subject of intensive study for more than three decades [1]. Initially the problem was studied to explain anomalous behavior of magnetic impurities embedded in a metallic host. Recent progress in the nano-fabrication technique of electronic devices enabled investigation of the Kondo effect by means of quantum dots (QD) in a very controllable way [2 [5]. The Kondo effect in the single electron transistor (SET) structures is characterized by an enhanced conductance due to the Kondo resonance [6 13]. The Kondo resonance at the Fermi level provides a new channel for electric current flowing through the QD. On the other hand, it is interesting to note that the Kondo scattering is a phasecoherent phenomenon which is characterized by a resonance phase shift of $\pi / 2$ with unitary tunneling cross section at low temperature and bias [6, [7]. The phase coherence of the Kondo scattering cannot be proved by the transport measurement in SET structures because the conductance through the QD measures only the probability of the transmission amplitude. In order to prove the phase-coherence one needs a device setup which includes quantum interference process. A typical example is a QD embedded in an Aharonov-Bohm interferometer [14 18] or in an Aharonov-Bohm ring [19 21], where the phase-coherence is studied through the dependence on the Aharonov-Bohm flux.

In this paper, we consider another type of simple quantum interference device with Kondo correlation: A perfect quantum wire with a side-coupled quantum dot (Fig.1(a)). This structure is reminiscent of T-shaped quantum wave guides known as electron stub tuners [22] in which the quasi-bound states in the stub play a major role in transport properties. On the other hand, we consider relatively weak tunneling limit between the wire and the dot. Therefore, both electron-electron interaction and quantum interference are important. In contrast to the SET geometry, transmission through the system consists of the interference between the ballistic channel and the resonant channel from the quantum dot, and the transport contains some informations on the phasecoherence through the quantum dot. We consider a simple one-dimensional quantum wire with a side-coupled Anderson impurity at site "0" (Fig.1(b)). The Hamiltonian of the system can be written as

$$
H=H_{0}+H_{D}+H_{T}
$$

where $H_{0}, H_{D}$, and $H_{T}$ represent the noninteracting wire, the interacting $\mathrm{QD}$, and tunneling, respectively, which are given by

$$
\begin{aligned}
H_{0} & =\sum_{k \sigma} \varepsilon_{k} c_{k \sigma}^{\dagger} c_{k \sigma}, \\
H_{D} & =\sum_{\sigma} \varepsilon_{d} d_{\sigma}^{\dagger} d_{\sigma}+U \hat{n}_{\uparrow} \hat{n}_{\downarrow}, \\
H_{T} & =-t^{\prime} \sum_{\sigma}\left(d_{\sigma}^{\dagger} c_{0 \sigma}+c_{0 \sigma}^{\dagger} d_{\sigma}\right) .
\end{aligned}
$$

Here $\varepsilon_{d}$ and $U$ represent the single-particle energy and the on-site Coulomb repulsion in the QD, respectively. $t^{\prime}$ denotes the tunneling matrix element between the QD level and the site "0" of the quantum wire. Note that the electron operator at site 0 is given by

$$
c_{0 \sigma}=\frac{1}{\sqrt{N}} \sum_{k} c_{k \sigma}
$$

where $N$ is the number of lattice sites that goes to infinity.

At low temperature and bias voltage, electron transport is dominated by the coherent transmission, and the linear-response conductance is given by the Landauertype formula

$$
G=\frac{2 e^{2}}{h} \int\left(-\frac{\partial f}{\partial \varepsilon}\right) T(\varepsilon) d \varepsilon,
$$


where $f$ and $T(\varepsilon)$ denote the Fermi distribution function and the transmission probability of an electron with energy $\varepsilon$, respectively. $T(\varepsilon)$ is related to the Green's function at site 0 with spin $\sigma, G_{0 \sigma}$ :

$$
T(\varepsilon)=\Gamma^{2}\left|G_{0 \sigma}(\varepsilon)\right|^{2},
$$

where $\Gamma$ corresponds to the coupling strength of the site 0 to the other part of the wire (which is proportional to the kinetic energy of the electrons in the wire). $G_{0 \sigma}$ can be rewritten in terms of the "exact" Green function of the dot, $G_{d \sigma}$,

$$
G_{0 \sigma}(\varepsilon)=\frac{1}{\varepsilon+i \Gamma}\left(1+\frac{t^{\prime 2}}{\varepsilon+i \Gamma} G_{d \sigma}(\varepsilon)\right) .
$$

At zero temperature only electrons at the Fermi level, $\varepsilon=0$, are important, and inelastic processes are fully suppressed. In this case the conductance is simplified as

$$
G=\frac{2 e^{2}}{h} T(0),
$$

and the dot Green's function $G_{d \sigma}$ satisfies the FriedelLangreth sum rule [23]:

$$
G_{d \sigma}(0)=\frac{1}{-\Gamma^{\prime} \cot \varphi+i \Gamma^{\prime}},
$$

where $\Gamma^{\prime}=t^{\prime 2} / \Gamma$ is the resonance width of the quantum dot level, and

$$
\varphi=\frac{\pi}{2} n_{d}
$$

with $n_{d}$ being the average occupation number of the QD.

By combining Eqs.(田), (哥), (6), and (7), one get the following very compact form of the zero-temperature conductance:

$$
G=\frac{2 e^{2}}{h} \cos ^{2} \varphi .
$$

The expression (9) is in good contrast with the case where the quantum dot is connected to two separate electrodes. In such a case, the zero-temperature conductance is given by $G=\frac{2 e^{2}}{h} \sin ^{2} \varphi$ for a symmetrically coupled junctions [6,7], and has maximum value in the Kondo limit, $n_{d} \simeq 1$. However, in our model, the conductance is fully suppressed in the Kondo limit. This is purely quantum effect associated with the Kondo correlation and can be understood in terms of the destructive interference between the ballistic channel and the Kondo resonance represented in the first and the second term of Eq.(5), respectively. On the other limit, $n_{d}=0$ or $n_{d}=2$, both spin and charge fluctuations are suppressed. Therefore, the coupling between the quantum dot and the wire becomes ineffective and the conductance approaches to the conductance quantum due to the ballistic transmission through the wire.
To study the problem in more detail, we adopt the slave-boson mean field theory (SBMFT) which is known to be a good approximation for describing the Fermiliquid properties with strongly renormalized parameters [1]. In the framework of the SBMFT for infinite $U$, the dot Green's function is given by

$$
G_{d \sigma}(\varepsilon)=\frac{1-n_{d}}{\varepsilon-\tilde{\varepsilon}_{d}+i\left(1-n_{d}\right) \Gamma^{\prime}},
$$

where the renormalized energy level $\tilde{\varepsilon}_{d}$ and the occupation number $n_{d}$ are to be obtained by solving the selfconsistent equations [1]:

$$
\begin{aligned}
\tilde{\varepsilon}_{d}-\varepsilon_{d} & =-\frac{2}{\pi} \frac{\partial}{\partial n_{d}} \int_{-D}^{D} f(\varepsilon) \tan ^{-1}\left[\frac{\left(1-n_{d}\right) \Gamma^{\prime}}{\tilde{\varepsilon}_{d}-\varepsilon}\right] d \varepsilon, \\
n_{d} & =-\frac{2}{\pi} \int f(\varepsilon) \Im \frac{1}{\varepsilon-\tilde{\varepsilon}_{d}+i\left(1-n_{d}\right) \Gamma^{\prime}} d \varepsilon .
\end{aligned}
$$

A constant density of states (DOS) for the wire at $-D<\varepsilon<D$ is assumed in the above equation for simplicity. At zero temperature the integrations in Eq.(11) can be performed analytically and we get

$$
\begin{aligned}
\tilde{\varepsilon}_{d}-\varepsilon_{d} & =-\frac{2 \Gamma^{\prime}}{\pi} \log \frac{\sqrt{\tilde{\varepsilon}_{d}^{2}+\left(1-n_{d}\right)^{2} \Gamma^{\prime 2}}}{D} \\
n_{d} & =\frac{2}{\pi} \tan ^{-1} \frac{\left(1-n_{d}\right) \Gamma^{\prime}}{\tilde{\varepsilon}_{d}} .
\end{aligned}
$$

A very important point is that the SBMFT satisfies automatically the unitarity of the scattering matrix, that is,

$$
R(\varepsilon)+T(\varepsilon)=1,
$$

where $R(\varepsilon)$ and $T(\varepsilon)$ denote the reflection and the transmission probability of an electron with energy $\varepsilon$, respectively. It should be noted that some other methods based on the large- $N_{s}$ approach $\left(N_{s}\right.$ being the spin degeneracy of the dot), such as the non-crossing approximation (NCA) or diagrammatic $1 / N_{s}$ expansion, do not satisfy this relation. In addition, Eqs. (5), (6), (10), and (12b) lead to the expression (9). That is, the zero-temperature conductance obtained by the SBMFT gives the exact expression of Eq.(9).

Fig.2 displays the conductance as a function of the quantum dot energy level. In the experiment $\varepsilon_{d}$ can be controlled by attaching a voltage gate to the QD. At zero temperature, the conductance shows a crossover from resonant reflection in the Kondo limit $\left(\varepsilon_{d} \ll-2 \Gamma^{\prime}\right)$ to perfect transmission where the coupling to the wire is ineffective $\left(\varepsilon_{d}>2 \Gamma^{\prime}\right)$. The perfect reflection in the Kondo limit originates from the completely destructive interference between the ballistic and the Kondo channel. At finite temperatures, the conductance shows a dip structure with an asymmetric line shape 24. The dip occurs, as a peak does for the Kondo effect in the SET 
structure, due to a continuous transition from the hightemperature Kondo phase to the low-temperature charge fluctuation phase. As the temperature increases the dip position moves toward $\varepsilon_{d}=0$ with reduced depth.

Fig. 3 explains the crossover behavior for a fixed temperature $T=0.1 \Gamma^{\prime}$, in relation to the Kondo temperature, $T_{K}$. At very low energy level $\left(\varepsilon_{d} \ll-2 \Gamma^{\prime}\right)$ the Kondo temperature is much smaller than the given temperature $T$. In this case the Kondo effect is negligible and the Kondo-resonant reflection is suppressed due to the ineffective coupling between the QD and the wire. By increasing $\varepsilon_{d}$, the Kondo temperature is increased exponentially and the Kondo effect becomes important. Then the current reaches its minimum at a point that satisfies the condition $T_{K}>T$ and $\varepsilon_{d}<-2 \Gamma^{\prime}\left(n_{d} \simeq 1\right)$. Further increasing $\varepsilon_{d}$ results in an increase of the current because of charge fluctuation. Kondo physics is no longer valid in this region and the transmission through the QD begins to resemble that of a pure quantum wire as $n_{d}$ decreases further, again due to the ineffective coupling.

In conclusion, we have analyzed the quantum transport through a ballistic quantum wire with a side-coupled interacting quantum dot. We have shown that the Kondo resonance associated with a quantum interference leads to a perfect reflection of the electrons. We have also discussed the anti-resonance behavior which originates from the crossover of the high temperature Kondo to the low temperature charge fluctuation region.

* Electronic address : kckang@phy0.chonbuk.ac.kr

[1] For a review, see. e.g., A. C. Hewson, The Kondo Problem to Heavy Fermions (Cambridge University Press, Cambridge 1993).

[2] D. Goldhaber-Gordon, H. Shtrikman, D. Abush-Magder, U. Meirav and M. A. Kastner, Nature 391, 156 (1998); D. Goldhaber-Gordon et al., Phys. Rev. Lett. 81, 5225 (1998).

[3] S. M. Cronenwett, T. H. Oosterkamp, and L. P. Kouwenhoven, Science 281, 540 (1998).

[4] J. Schmid et al., Physica 256B-258B, 182 (1998).

[5] F. Simmel et al., Phys. Rev. Lett. 83,804 (1999).

[6] L. I. Glazman and M. E. Raikh, Pis'ma Zh. Eksp. Teor. Fiz. 47, 378 (1988) [JETP Lett. 47, 452 (1988)].

[7] T. K. Ng and P. A. Lee, Phys. Rev. Lett. 61, 1768 (1988).

[8] S. Hershfeld, J. H. Davies and J. W. Wilkins, Phys. Rev. Lett. 67, 3720 (1991); Phys. Rev. B 46, 7046 (1992).

[9] Y. Meir, N. S. Wingreen and P. A. Lee, Phys. Rev. Lett. 70, 2601 (1993); N. S. Wingreen and Y. Meir, Phys. Rev. B 49, 11040 (1994).

[10] A. L. Yeyati, A. Martín-Rodero and F. Flores, Phys. Rev. Lett. 71, 2991 (1993).

[11] J. König, H. Schoeller and G. Schön, Phys. Rev. Lett. 76, 1715 (1996); J. König, J. Schmid, H. Schoeller and
G. Schön, Phys. Rev. B 54, 16820 (1996).

[12] K. Kang, Phys. Rev. B 58, 9641 (1998); S. Y. Cho, K. Kang, and C.-M. Ryu, Phys. Rev. B 60, 16874 (1999).

[13] L. Craco and K. Kang, Phys. Rev. B 59, 12244 (1999).

[14] C. Bruder, R. Fazio, and H. Schoeller, Phys. Rev. Lett. 76, 114 (1996).

[15] M. A. Davidovich, E. V. Anda, J. R. Iglesias, and G. Chiappe, Phys. Rev. B 55, R7335 (1997).

[16] W. Izumida, O. Sakai, and Y. Shimizu, J. Phys. Soc. Jpn 66, 717 (1997).

[17] U. Gerland, J. von Delft, T. A. Costi, and Y. Oreg, Phys. Rev. Lett. 84, 3710 (2000).

[18] Y. Ji, M. Heiblum, D. Sprinzak, D. Mahalu, and H. Shtrikman, unpublished (cond-mat/0007332).

[19] V. Ferrari, G. Chiappe, E. V. Anda, and M. A. Davidovich, Phys. Rev. Lett. 82, 5088 (1999).

[20] K. Kang and S.-C. Shin, cond-mat/9912399 (unpublished).

[21] H.-P. Eckle, H. Johannesson, and C. A. Stafford, J. Low Temp. Phys. 118, 475 (2000).

[22] P. Debray, O. E. Raichev, P. Vasilopoulos, M. Rahman,R. Perrin, and W. C. Mitchell, Phys. Rev. B 61, 10950 (2000), and references therein.

[23] D. C. Langreth, Phys. Rev. 150, 516 (1966).

[24] A similar dip structure has been observed in a SET experiment by Göres et al., Phys. Rev. B 62, 2188 (2000). However it is not clear in their experiment if the antiresonance is associated with the Kondo effect.

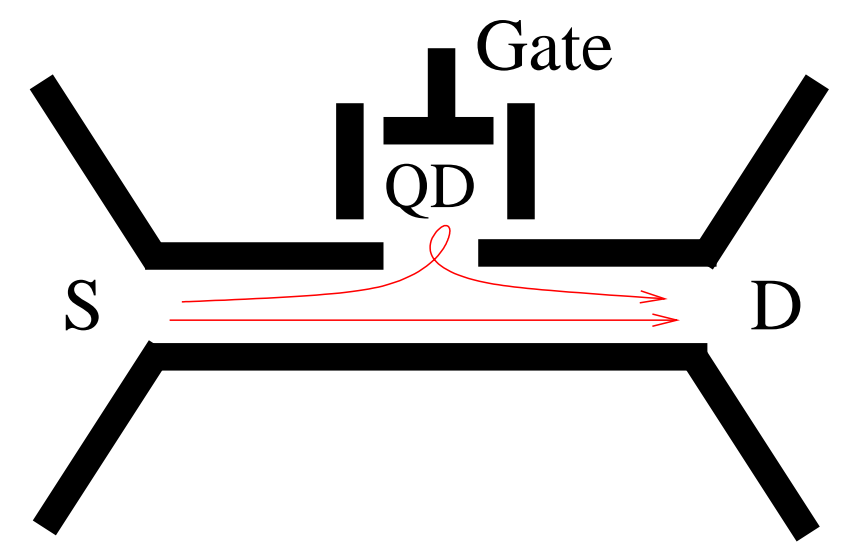

(a)

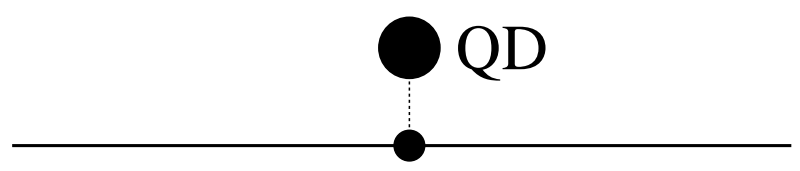

(b) 
FIG. 1. (a) Schematic diagram of the quantum wire with a side-coupled quantum dot. (b) Model of the device for the quantum wire and a side-attached quantum dot. The quantum wire is considered to be impurity-free one-dimensional metal and the quantum dot is modeled as an Anderson impurity.

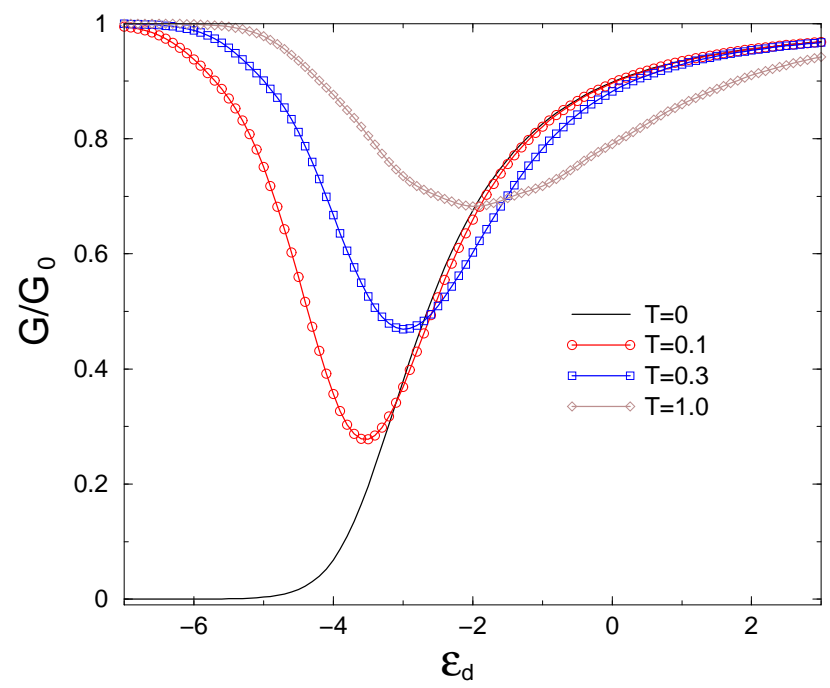

FIG. 2. Conductance (in unit of $G_{0}=2 e^{2} / h$ ) as a function of the level position of the quantum dot $\left(\varepsilon_{d}\right)$ for four different temperatures. All the energy scale is renormalized in unit of $\Gamma^{\prime}$ in this figure and in Fig. 3.

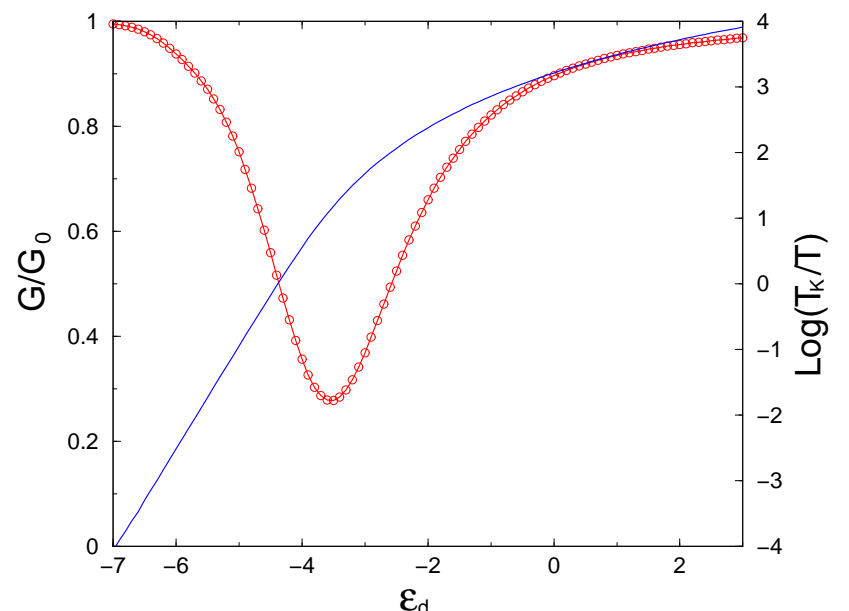

FIG. 3. Conductance (solid line with circles) and Kondo temperature in log scale (solid line), as a function of the level position of the quantum dot for $T=0.1 \Gamma^{\prime}$. 